\title{
Existe associação entre os desconfortos no puerpério imediato e a via de parto? Um estudo observacional
}

\author{
Is there association between the discomforts of the immediate postpartum \\ period and type of delivery? An observational study
}

Thalita Rodrigues Christovam Pereira`, Fábio Tadeu Montesano², Pryscilla Dieguez Ferreira ${ }^{3}$, Andrea Simões Minozzi Ana Carolina Sartorato Beleza ${ }^{5}$

'Programa de Pós-graduação Interdisciplinar em Ciências da Saúde, Universidade Federal de São Paulo (UNIFESP) - Santos (SP), Brasil.

${ }^{2}$ Setor de Estatística, UNIFESP - Santos (SP), Brasil.

${ }^{3}$ Women and Men's Health Physiotherapy Department, James Paget University Hospital - Great Yarmouth, Inglaterra.

${ }^{4}$ Departamento de Obstetrícia, Fisioterapia em Obstetrícia, UNIFESP - São Paulo (SP), Brasil.

5Universidade Federal de São Carlos (UFSCar) - São Carlos (SP), Brasil.

DOI: http://dx.doi.org/10.7322/abcshs.v42i2.1007

\section{RESUMO}

Introdução: A via de parto pode ser um fator determinante para o desenvolvimento de desconfortos no puerpério imediato. Objetivo: Identificar os desconfortos referidos pelas mulheres no puerpério imediato e compará-los entre as submetidas ao parto vaginal e à cesárea. Métodos: Estudo analítico transversal desenvolvido em uma maternidade pública com levantamento de prontuários que continham informações da avaliação fisioterapêutica de puérperas atendidas no período de fevereiro a novembro de 2011. Resultados: Foram incluídos 346 prontuários neste estudo. Queixas do puerpério imediato: dor abdominal $(31,5 \%)$, edema $(19,1 \%)$, lombalgia $(18,2 \%)$, cervicalgia $(9,8 \%)$ e desconforto em membros inferiores (5,8\%). Cesárea foi a via de parto que acarretou maior desconforto, relacionando-se à presença de dor abdominal $(p=0,001)$, cervicalgia $(p=0,017)$ e edema $(p=0,017)$. Conclusão: Os achados sugerem que as mulheres que passam por cesárea estão vulneráveis à maior morbidade durante o puerpério imediato em comparação ao parto vaginal.

Palavras-chave: período pós-parto; saúde da mulher; fisioterapia.

\begin{abstract}
Introduction: The type of delivery may be a determining factor for the development of discomfort in the immediate postpartum period. Objective: To identify the discomfort related by women in the immediate postpartum period and to compare them among those submitted to vaginal delivery and cesarean Methods: Cross-sectional analytical study developed in a public maternity hospital with medical records that contained information of physical therapy evaluation of postpartum women attended from February to November 2011. Results: A total of 346 records were included in this study. Immediate postpartum complaints: abdominal pain $(31.5 \%)$, edema (19.1\%), low back pain (18.2\%), neck pain $(9.8 \%)$ and discomfort in the lower limbs (5.8\%). Cesarean was the mode of delivery that caused greater discomfort related to the presence of abdominal pain $(p=0.001)$, neck pain $(p=0.017)$ and edema $(p=0.017)$. Conclusion: The findings suggest that women who undergo cesarean are vulnerable to greater morbidity compared to vaginal delivery during the immediate postpartum period.
\end{abstract}

Keywords: postpartum period; women's health; physical therapy specialty.

Recebido em: 11/11/2016

Revisado em: 26/03/2017

Aprovado em: 04/04/2017

Autor para correspondência: Thalita Rodrigues Christovam Pereira - Rua Silva Jardim, 136 - Vila Mathias - CEP: 11015-020 - Santos (SP), Brasil -

Fonte de financiamento: Conselho Nacional de Desenvolvimento Científico e Tecnológico (CNPq). 


\section{INTRODUÇÃO}

O puerpério é marcado por grandes mudanças nos âmbitos físico, emocional e social tanto para as mulheres quanto para seus familiares ${ }^{1}$. Trata-se de um período que, embora fisiológico, apresenta predisposição para o desenvolvimento de desconfortos que podem comprometer a qualidade de vida da mulher, assim como favorecer o aparecimento de complicações devido a diversas alterações morfológicas e hormonais presentes nessa fase $\mathrm{e}^{2}$ e por isso merecem investigação.

Embora a Organização Mundial da Saúde (OMS) tenha proposto melhorar a saúde materna entre os Objetivos de Desenvolvimento do Milênio ${ }^{3}$, ainda há pouco na literatura científica sobre os cuidados oferecidos à puérpera, especialmente em relação à atuação da Fisioterapia. A discrepância entre o modelo assistencial preconizado pelas organizações de saúde e a realidade presente nos serviços demonstra desconhecimento das reais necessidades das mulheres no período puerperal por parte dos profissionais da saúde.

A prestação de cuidado no puerpério é de vital importância para o bem-estar da mulher. A via de parto e a atenção dispensada ao puerpério imediato podem contribuir para o aparecimento de desconfortos que não estavam presentes no período gestacional ou mesmo agravar de queixas preexistentes. Estudo sobre o puerpério ${ }^{4}$ apontou que a dor na cicatriz abdominal era a queixa predominante em puérperas provenientes de cesárea, enquanto a dor pélvica era apresentada por puérperas que passaram pelo parto vaginal.

Desconfortos e dores no puerpério podem representar fatores limitantes ao autocuidado e ao cuidado com o recém-nascido. Dessa forma, investigar a relação entre a via de parto e predisposição para o aparecimento de determinadas queixas puerperais busca fundamentar as condutas de cuidado em saúde baseadas em evidências, bem como estimular a adoção de práticas preventivas na atenção à saúde da puérpera.

Assim, os objetivos deste estudo foram identificar os desconfortos referidos no puerpério imediato e comparar tais desconfortos entre mulheres submetidas ao parto vaginal e à cesárea.

\section{MÉTODOS}

Este estudo analítico transversal foi conduzido de forma retrospectiva utilizando prontuários de mulheres que receberam assistência fisioterapêutica durante o puerpério imediato em uma maternidade pública de um Hospital Escola localizado no litoral paulista.

O estudo foi aprovado pelo Comitê de Ética em Pesquisa da Universidade Federal de São Paulo (CEP/UNIFESP) (nº 296.671) e pelo Comitê de Ética da instituição na qual o estudo foi desenvolvido ( $\mathrm{n}^{\circ}$ 006/2010). Por se tratar de uma pesquisa retrospectiva em prontuários, o uso de Termo de Consentimento Livre e Esclarecido (TCLE) foi dispensado.

A coleta de dados ocorreu por meio de levantamento dos prontuários que continham informações sobre a avaliação fisioterapêutica de puérperas atendidas na maternidade no período de fevereiro a novembro de 2011. Uma comissão foi responsável por selecionar quais fichas deveriam ser incluídas e quais seriam excluídas do estudo. Os critérios de inclusão foram: prontuários de puérperas com idade igual ou superior a 18 anos e com recém-nascido único. As fichas de avaliação de puérperas com idade inferior a 18 anos e com conteúdo incompleto foram excluídas do estudo.

As seguintes informações foram coletadas:

- dados pessoais: idade, escolaridade e estado civil;

- dados da gestação atual: se houve acompanhamento médico durante a gestação, número de consultas pré-natal, queixas durante o período gestacional;

- via de parto; e

- desconfortos apresentados no puerpério.

O programa estatístico R versão 3.1.3 (R Development Core Team, Viena, Áustria) foi utilizado para análise descritiva dos dados, seguida de análise inferencial com o teste do $\chi^{2}$ de Pearson para comparação entre as vias de parto e os desconfortos no puerpério. O nível de significância considerado foi de $5 \%(\mathrm{p}<0,05)$.

\section{RESULTADOS}

Dentre as 365 fichas de avaliações fisioterapêuticas disponíveis nos prontuários, 19 foram excluídas por não obedecerem aos critérios de inclusão, obtendo-se, portanto, 346 para a análise (Figura 1).

Neste estudo, 55,8\% ( $n=193)$ das mulheres passaram por cesárea, enquanto $44,2 \%(n=153)$ tiveram seus filhos por via vaginal. As características gerais da amostra segundo a via de parto podem ser observadas na Tabela 1.

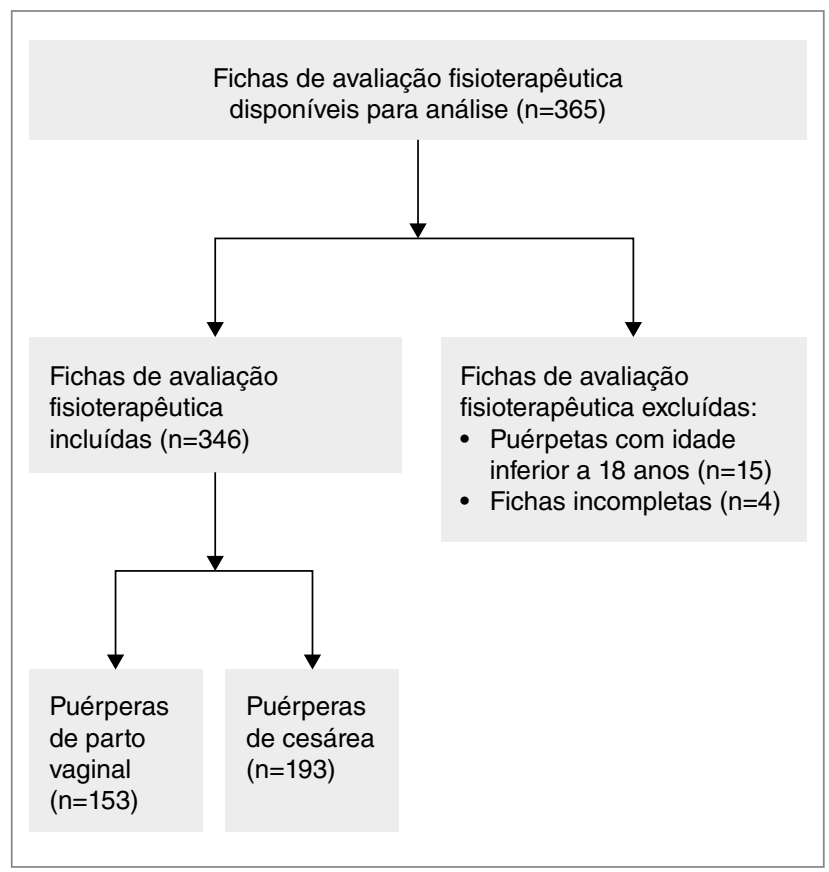

Figura 1: Fluxograma representativo da seleção das fichas de acordo com os critérios de inclusão e exclusão 
$\mathrm{Na}$ avaliação fisioterapêutica no puerpério imediato, 72,8\% $(\mathrm{n}=252)$ dessas mulheres se queixaram de algum desconforto, sendo $31,5 \%(n=109)$ dor abdominal, 19,1\% ( $n=66)$ edema, $18,2 \%$ $(n=63)$ lombalgia, 9,8\% ( $n=34)$ cervicalgia e 5,8\% ( $n=20)$ desconfortos em membros inferiores.

A comparação entre as vias de parto em relação aos desconfortos puerperais (Tabela 2) permite afirmar que houve associação entre as vias de parto e as variáveis: dor abdominal $(\mathrm{p}=0,001)$, cervicalgia $(\mathrm{p}=0,017)$ e edema $(\mathrm{p}=0,017)($ Tabela 2$)$.

\section{DISCUSSÃO}

A cesárea foi a via de parto que acarretou maior frequência de queixas por parte das puérperas, sobretudo quando se trata da presença de dor abdominal, cervicalgia e edema durante o puerpério imediato.

A dor abdominal no puerpério pode estar ligada a diversos fatores. Durante a amamentação ocorre liberação de ocitocina e

Tabela 1: Características gerais da amostra segundo a via de parto

\begin{tabular}{|l|c|c|}
\hline Características & $\begin{array}{c}\text { Vaginal } \\
(\mathbf{n = 1 5 3})\end{array}$ & $\begin{array}{c}\text { Cesárea } \\
(\mathbf{n}=193)\end{array}$ \\
\hline Idade (anos), média $\pm \mathrm{DP}$ & $25,0 \pm 6,5$ & $26,4 \pm 6,2$ \\
\hline Estado civil, $\mathrm{n}(\%)$ & & \\
\hline Solteira & $52(33,9)$ & $58(30,0)$ \\
\hline Casada & $47(30,7)$ & $77(39,9)$ \\
\hline União estável & $44(28,8)$ & $50(25,9)$ \\
\hline Viúva & - & $1(0,5)$ \\
\hline Escolaridade, $\mathrm{n}(\%)$ & & \\
\hline Ensino fundamental incompleto & $15(9,8)$ & $12(6,2)$ \\
\hline Ensino fundamental completo & $18(11,8)$ & $10(5,2)$ \\
\hline Ensino médio incompleto & $17(11,1)$ & $15(7,8)$ \\
\hline Ensino médio completo & $49(32,0)$ & $78(40,4)$ \\
\hline Ensino técnico incompleto & - & - \\
\hline Ensino técnico completo & $3(2,0)$ & - \\
\hline Ensino superior incompleto & $2(1,3)$ & $4(2,1)$ \\
\hline Ensino superior completo & $3(2,0)$ & $10(5,2)$ \\
\hline Acompanhamento pré-natal, $\mathrm{n}(\%)$ & $149(97,4)$ & $192(99,5)$ \\
\hline Consultas pré-natal, média $\pm D P$ & $8,1 \pm 2,3$ & $8,7 \pm 2,6$ \\
\hline Queixas gestacionais, $\mathrm{n}(\%)$ & & \\
\hline Edema & $82(53,6)$ & $118(61,1)$ \\
\hline Lombalgia & $94(61,4)$ & $122(63,2)$ \\
\hline Câimbra & $90(58,8)$ & $116(60,1)$ \\
\hline Parestesia & $39(25,5)$ & $59(30,6)$ \\
\hline DP: & &
\end{tabular}

DP: desvio padrão; n: número da amostra; \%: frequência (porcentagem).

Tabela 2: Comparação entre a via de parto e os desconfortos puerperais durante o período de internação

\begin{tabular}{|l|c|c|c|}
\hline $\begin{array}{l}\text { Queixas } \\
\text { puerperais, } \mathbf{n}(\%)\end{array}$ & $\begin{array}{l}\text { Vaginal } \\
(\mathbf{n = 1 5 3 )}\end{array}$ & $\begin{array}{l}\text { Cesárea } \\
(\mathbf{n = 1 9 3 )}\end{array}$ & Valor $\mathbf{p}$ \\
\hline Cervicalgia & $8(5,2)$ & $26(13,5)$ & $0,017^{*}$ \\
\hline Lombalgia & $31(20,3)$ & $32(16,6)$ & 0,459 \\
\hline Dor abdominal & $31(20,3)$ & $78(40,4)$ & $0,001^{*}$ \\
\hline Edema & $20(13,1)$ & $46(23,8)$ & $0,017^{*}$ \\
\hline $\begin{array}{l}\text { Desconfortos em } \\
\text { membros inferiores }\end{array}$ & $8(5,2)$ & $12(6,2)$ & 0,873 \\
\hline
\end{tabular}

${ }^{*} \mathrm{p}<0,05$ : estatisticamente significativo, teste do $\chi^{2}$; DP: desvio padrão; n: número da amostra; \%: frequência (porcentagem). aumento do tônus uterino, levando ao aumento das cólicas abdominais, independentemente da via de parto ${ }^{5}$. Em relação à cesárea, as queixas abdominais somam-se ao desconforto inerente à incisão cirúrgica ${ }^{6}$. Puérperas de cesárea tendem a maiores compensações para amamentar, uma vez que a presença da ferida cirúrgica limita a mudança de posição e a adoção de posturas adequadas para o aleitamento ${ }^{5}$, favorecendo o aparecimento de cervicalgia e lombalgia.

Neste estudo, a presença de queixa de lombalgia foi semelhante entre puérperas provenientes de parto vaginal e de cesárea. A lombalgia no puerpério pode apresentar diversos fatores predisponentes. A diástase dos músculos retos abdominais, desencadeada na gestação, compromete a capacidade da musculatura abdominal de estabilização do tronco, interferindo no alinhamento postural e no equilíbrio da musculatura lombar ${ }^{7}$, bem como nas atividades do cotidiano. Assim como a diástase dos músculos retos abdominais, a incisão cirúrgica da cesárea também contribui para a redução da ação da musculatura abdominal como estabilizadora de tronco, contribuindo para susceptibilidade à lombalgia.

Dependendo do nível álgico apresentado, a lombalgia pode resultar em variados graus de incapacidade motora ${ }^{8}$. Dessa forma, é importante que seja identificada e classificada no início do período pós-parto ${ }^{8}$ com avaliação física por profissional da saúde capacitado, possibilitando, assim, que as intervenções terapêuticas possam ser direcionadas para as queixas das puérperas o mais precocemente possível.

A cicatriz cirúrgica abdominal em puérperas de cesárea pode dificultar a via de drenagem pelo sistema linfático e favorecer restrição de movimentação por dor e/ou medo, levando à maior permanência no leito e predispondo ao surgimento de edema em membros inferiores. Estudos randomizados e controlados se fazem necessários para investigação da relação entre os fatores aqui levantados e o edema no puerpério de cesárea.

A cesárea foi o procedimento mais praticado dentre as mulheres incluídas no presente estudo. Esse achado está de acordo com a literatura, a qual mostra que, apesar da recomendação da OMS, as taxas de cesáreas vêm aumentando em muitos países, dentre eles o Brasil ${ }^{9,10}$. A crescente demanda por cesárea parece estar baseada na crença de que a qualidade da atenção obstétrica encontra-se intimamente associada à tecnologia no trabalho de parto e no nascimento ${ }^{11}$.

Sabe-se que as taxas de cesáreas estão relacionadas a fatores socioculturais, demográficos e econômicos ${ }^{12}$. Informações vinculadas na mídia sobre o processo de parturição, novas tecnologias, inserção da mulher no mercado de trabalho, fatores relacionados ao modelo assistencial desenvolvido nos países, disponibilidade da equipe médica, subdiagnóstico de riscos maternos e fetais ${ }^{13}$, entre diversos outros exemplos, podem ser facilmente reconhecidos na prática clínica.

Quanto ao parto vaginal, a ausência de especificação nos prontuários sobre a presença de laceração e/ou episiotomia é considerada uma limitação para este estudo, impossibilitando uma 
análise mais aprofundada. As limitações funcionais impostas pela presença de laceração espontânea e episiotomia podem interferir na qualidade de vida e na sexualidade pós-parto.

A escolha da via de parto por parte das mulheres está permeada por desejos de garantir a saúde materna e fetal, evitar a dor e o sofrimento durante o puerpério ${ }^{14}$. Diferentemente da crença popular na vivência de menor morbidade puerperal, a literatura tem demonstrado maiores riscos de susceptibilidade à redução da saúde física ${ }^{15,16}$ e emocional das mulheres após cesárea ${ }^{17}$.

A morbidade materna pode ser uma condição tanto temporária quanto permanente e, assim, apresenta-se com difícil mensuração, visto que a percepção da morbidade varia entre as diferentes culturas ${ }^{3}$. Os desconfortos presentes são fatores que contribuem para limitação da movimentação das puérperas, dificultando as atividades cotidianas de autocuidado e cuidado com o bebê, favorecendo a ocorrência de complicações. Por consequência, tais complicações acarretarão ao sistema de saúde maiores custos com medicamentos, tratamentos e internações para essas mulheres.

A investigação dos sintomas físicos no puerpério possibilita a adoção de estratégias para a recuperação plena da mulher. Importantes fatores devem ser considerados em relação à qualidade da assistência obstétrica, tanto no âmbito privado quanto público. Destacam-se o uso de medicalização excessiva em cuidados obstétricos, prática rotineira de episiotomia e baixo uso de métodos não farmacológicos para o alívio da dor $^{18}$. Além de afetar a saúde da mulher, a morbidade materna apresenta forte elo com a saúde neonatal ${ }^{3}$, potencializando-se como fator de susceptibilidade para o insucesso no aleitamento materno ${ }^{19}$.

Assim, ressalta-se a necessidade da ampliação da prática interdisciplinar em saúde e a promoção da humanização no cuidado materno-infantil. A Fisioterapia, especialmente na área de atenção à saúde da mulher, dispõe de recursos não farmacológicos para o alívio dessas queixas e prevenção de complicações, tais como cinesioterapia, crioterapia ${ }^{20}$ e eletroterapia ${ }^{18,21}$.
A atuação desse profissional da saúde pode ocorrer desde a gestação ${ }^{22}$, de forma que a preparação do organismo feminino e a educação em saúde contribuam para o empoderamento das mulheres acerca das transformações em seu corpo provenientes do processo gestacional; durante o trabalho de parto $^{23}$, com o uso de posicionamentos e recursos físicos para proporcionar conforto e alívio da dor; e no puerpério ${ }^{18,21}$, quando a involução do organismo às condições pré-gravídicas, o aleitamento materno e as tarefas cotidianas favorecem o aparecimento de queixas de morbidade.

A inserção de técnicas não farmacológicas de domínio fisioterapêutico no ambiente hospitalar pode contribuir, ainda, para a diminuição dos custos em relação ao tempo de internação, visto que esses recursos tendem a se apresentar menos elevados em comparação à administração de fármacos, e por vezes são capazes de fornecer alívio e conforto desejados, assim como mínimos efeitos adversos ${ }^{20}$.

No contexto geral, a implementação de políticas de saúde que estimulem e favoreçam o parto vaginal, diminuindo, assim, as taxas de cesáreas, também pode contribuir para a redução da morbidade puerperal.

Os desconfortos referidos pelas mulheres no puerpério imediato foram: dor abdominal, edema, lombalgia, cervicalgia e desconforto em membros inferiores. Os achados sugerem que as mulheres que passam por cesárea estão mais vulneráveis à maior morbidade do que as de parto vaginal durante o puerpério imediato. Cuidados dispensados às puérperas na maternidade devem levar em consideração a via de parto e o comprometimento da mobilidade visando restaurar a funcionalidade.

\section{AGRADECIMENTOS}

À Santa Casa de Misericórdia de Santos (ISCMS) e ao Departamento de Ciências do Movimento Humano (DCMH) da Universidade Federal de São Paulo (UNIFESP) pelo apoio institucional ao desenvolvimento da pesquisa.

\section{REFERÊNCIAS}

1. Haran C, Van Driel M, Mitchell BL, Brocribb WE. Clinical guidelines for postpartum women and infants in primary care - a systematic review. BMC Pregnancy Childbirth. 2014;14:51. http://dx.doi.org/10.1186/1471-2393-14-51

2. Lavand'homme P. Chronic pain after childbirth. Curr Opin Anesthesiol. 2013;26(3):273-7. http://dx.doi.org/10.1097/ACO.0b013e328360c57b

3. Vanderkuik RC, Tunçalp Ö, Chou D, Say L. Framing maternal morbidity: WHO scoping exercise. BMC Pregnancy Childbirth. 2013;13:213. http://dx.doi.org/10.1186/1471-2393-13-213

4. Eisenach J, Pan P, Smiley RM, Lavand'homme P, Landau R, Houle TT. Resolution of pain after childbirth. Anesthesiol. 2013;118(1):143-51. http://dx.doi.org/10.1097/ALN.0b013e318278ccfd
5. Wen L, Hilton G, Carvalho B. The impact of breastfeeding on postpartum pain after vaginal and cesarean delivery. J Clin Anesth. 2015;27(1):33-8.

http://dx.doi.org/10.1016/.j.jclinane.2014.06.010

6. Hardy-Fairbanks AJ, Lauria MR, Mackenzie T, McCarthy Jr. $M$. Intensity and unpleasantness of pain following vaginal and cesarean delivery: a prospective evaluation. Birth. 2013:40(2):125-33.

http://dx.doi.org/10.1111/birt.12039

7. Sperstad JB, Tennfjord MK, Hilde G, Ellström-Engh M, Bø K Diastasis recti abdominis during pregnancy and 12 mounths after childbirth: prevalence, risk factors and report of lumbopelvic pain. Br J Sports Med. 2016;50:1092-6.

http://dx.doi.org/10.1136/bjsports-2016-096065 
8. Olsson CB, Grooten WJA, Nilsson-Wikmar L, Harms-Ringdahl $\mathrm{K}$, Lundberg M. Catastrophizing during and after pregnancy: associations with lumbopelvic pain and postpartum physical ability. Phys Ther. 2012;92(1):49-57. http://dx.doi.org/10.2522/ptj.20100293

9. Souza JP, Betran AP, Dumont A, de Mucio B, Gibbs Pickens CM, Deneux-Tharaux $C$, et al. A global reference for caesarean section rates (C-Model): a multicountry cross-sectional study. BJOG. 2016;123(3):427-36 http://dx.doi.org/10.1111/1471-0528.13509

10. Khunpradit S, Tavender E, Lumbiganon P, Laopaiboon M, Wasiak J, Gruen R. Non-clinical interventions for reducing unnecessary caesarean section. Cochrane Database Syst Rev. 2011;6:CD005528. http://dx.doi.org/10.1002/14651858.CD005528.pub2

11. do Carmo Leal M, da Silva AA, Dias MA, da Gama SG, Rattner D, Moreira ME, et al. Birth in Brazil: national survey into labour and birth. Reprod Health. 2012;9:15. http://dx.doi.org/10.1186/1742-4755-9-15

12. d'Orsi E, Brüggermann OM, Diniz CSG, Aguiar JM, Gusman CR, Torres JA, et al. Desigualdades sociais e satisfação das mulheres com o atendimento ao parto no Brasil: estudo nacional de base hospitalar. Cad Saúde Pública. 2014;30(Supl. 1):S154-68. http://dx.doi.org/10.1590/0102-311X00087813

13. Ji H, Jiang H, Yang L, Qian X, Tang S. Factors contributing to the rapid rise of caesarean section: a prospective study of primiparous Chinese women in Shanghai. BMJ Open. 2015;5(11):e008994 http://dx.doi.org/10.1136/bmjopen-2015-008994

14. Benute GRG, Nomura RY, Santos AM, Zarvos MA, Lucia MCS, Francisco RPV. Preferência pela via de parto: uma comparação entre gestantes nulíparas e primíparas. Rev Bras Ginecol Obstet. 2013;35(6):281-5.

http://dx.doi.org/10.1590/S0100-72032013000600008

15. Woolhouse H, Perlen S, Gartland D, Brown SJ. Physical health and recovery in the first 18 months postpartum: does cesarean section reduce long-term morbidity? Birth. 2012;39(3):221-9. http://dx.doi.org/10.1111/j.1523-536X.2012.00551.x
16. Rowlands IJ, Redshaw M. Mode of birth and women's psychological and physical wellbeing in the postnatal period. BMC Pregnancy Childbirth. 2012;12:138. http://dx.doi.org/10.1186/1471-2393-12-138

17. Kuo S-Y, Chien S-R, Tzeng Y-L. Depression and anxiety trajectories among women who undergo an elective cesarean section. PLoS One. 2014;9(1):e86653 http://dx.doi.org/10.1371/journal.pone.0086653

18. Pitangui ACR, Araújo RC, Bezerra MJS, Robeiro CO, Nakano AMS. Low and high-frequency TENS in post-episiotomy pain relief: a randomized, double-blind clinical trial. Braz J Phys Ther. 2014;18(1):72-8.

http://dx.doi.org/10.1590/S1413-35552012005000143

19. Stuebe AM, Horton BJ, Chetwynd E, Watkinds S, Grewen K, Meltzer-Brody S. Prevalence and risk factors for early, undesired weaning attributed to lactation dysfunction. J Womens Health (Larchmt). 2014;23(5):404-12. http://dx.doi.org/10.1089/jwh.2013.4506

20. Francisco AA, Oliveira SMJV, Leventhal LC, Bosco CS. Crioterapia no pós-parto: tempo de aplicação e mudanças na temperatura perineal. Rev Esc Enferm USP. 2013;47(3):555-61. http://dx.doi.org/10.1590/S0080-623420130000300005

21. Kayman-Kose S, Arioz DT, Toktas H, Koken G, Kanat-Pektas M, Kose M, et al. Transcutaneous electrical nerve stimulation (TENS) for pain control after vaginal delivery and cesarean section. $J$ Mater Fetal Neonatal Med. 2014:27(15):1572-5. http://dx.doi.org/10.3109/14767058.2013.870549

22. Progianti JM, Costa RF. Práticas educativas desenvolvidas por enfermeiras: repercussões sobre vivências de mulheres na gestação e no parto. Rev Bras Enferm. 2012;65(2):257-63. http://dx.doi.org/10.1590/S0034-71672012000200009

23. Castro AS, Castro AC, Mendonça AC. Abordagem fisioterapêutica no pré-parto: proposta de protocolo e avaliação da dor. Fisioter Pesqui. 2012;19(3):210-4. http://dx.doi.org/10.1590/S1809-29502012000300004 